\title{
Fatal Acute Liver Failure as a Consequence of Regorafenib Treatment in a Metastatic Colon Cancer
}

\author{
Dominique Béchade $^{a} \quad$ Marie Desjardin ${ }^{a} \quad$ Claire Castain $^{b}$ \\ Pierre-Henri Bernard ${ }^{c}$ Marianne Fonck ${ }^{a}$ \\ ${ }^{a}$ Digestive Tumors Unit, Institut Bergonié, Bordeaux, France; \\ ${ }^{b}$ Pathology Service, CHU de Bordeaux, Bordeaux, France; \\ ${ }^{c}$ Hepato-Gastroenterology Service, CHU de Bordeaux, Bordeaux, France
}

\section{Keywords}

Liver failure $\cdot$ Metastatic colorectal cancer $\cdot$ Regorafenib

\begin{abstract}
Regorafenib is a multikinase inhibitor which showed benefits in pretreated metastatic colorectal cancer patients. Hepatotoxicity has been described as a frequent side effect. We report the case of a 65 -year-old patient presenting with jaundice, fever, and hepatocellular insufficiency which led to death of the patient. She had previously been treated with several lines of chemotherapy for sub- and diaphragmatic ganglionic metastases of a colon adenocarcinoma. There were no liver metastases. The fatal liver failure occurred at the beginning of treatment with regorafenib at a dosage of 3 tablets per day. No concomitant treatment was given, and other causes of liver damage were eliminated. The liver biopsy showed hepatocyte necrosis with lymphocyte infiltration. This observation illustrates the risk of severe hepatic involvement typically occurring within the first 2 months of treatment. Monitoring liver biology every 2 weeks is essential during the first 2 months to detect any hepatotoxicity.
\end{abstract}


 Oncology}

\section{Introduction}

Regorafenib is an oral multi-tyrosine kinase inhibitor (TKI) that is indicated as monotherapy for the treatment of advanced colorectal cancer (CORRECT trial) [1] and gastrointestinal stromal tumors after imatinib and sunitinib failure (GRID trial) [2]. This TKI has a potent inhibitory activity against vascular endothelial growth factor receptors 1-3 (VEGFR1, 2, and 3), PDGFR- $\beta$, FGFR1, RAF-1, and the mutant oncogenic kinases KIT, RET, and BRAF [3].

The risk of TKI hepatotoxicity was clearly identified in two meta-analyses [4, 5], possibly favored by underlying viral or drug hepatopathies. Furthermore, fatal hepatocellular insufficiency has been reported with several TKIs [6]. Abnormalities in liver function tests can be very common among patients receiving regorafenib [7], and these biological abnormalities usually appear fairly early following treatment initiation, typically in the first 2 cycles [8]. Severe drug-induced liver injury with fatal outcome occurred in $0.3 \%$ of 1,100 regorafenibtreated patients across all published clinical trials. In the phase III CORRECT trial [1], fatal hepatic failure occurred in $1.6 \%$ of the patients of the regorafenib arm and in $0.4 \%$ of the placebo arm; all the patients with hepatic failure had metastatic disease in the liver. In the phase III GRID trial [2], fatal hepatic failure occurred in $0.8 \%$ of the patients of the regorafenib arm. In the CONCUR Asian trial [9], hepatotoxicity was more frequently associated with a cytolysis syndrome than in the CORRECT trial (elevated alanine aminotransferase [ALT] in $7 \%$ of the cases and elevated aspartate aminotransferase in $6 \%$ of the cases). Similar data were observed in the CORRECT trial adapted to a Japanese population and a non-Japanese population, with a significantly higher rate of liver toxicity in the former [10].

Here, we report a case of liver toxicity at a subtherapeutic dosage of regorafenib and discuss the potential mechanisms involved.

\section{Case Presentation}

In 2011, a 65-year-old patient with a lieberkühnian colonic adenocarcinoma was treated by surgery followed by 6 months of adjuvant chemotherapy with FOLFOX85. Supra- and subdiaphragmatic lymph node metastasis that had appeared in June 2012 warranted chemotherapy with FOLFIRI-cetuximab. The treatment was complicated by the appearance of Takotsubo syndrome, imposing the stoppage of 5-fluorouracil. Treatment was picked up again with irinotecan and cetuximab. A year later, a posterior mediastinal and supraclavicular lymph node relapse prompted the resumption of treatment with raltitrexed and irinotecan. In October 2014, nonfebrile diarrhea was associated with a predominant ALT cytolysis syndrome, 7 times the upper limit of normal $(7 \times \mathrm{N})$, with no hepatocellular insufficiency. Hepatobiliary ultrasound was normal. Serology was negative for hepatitis A, B, C, and E; the saturation coefficient of transferrin was normal. Uridine diphosphate glucuronosyltransferase $1 \mathrm{~A} 1$ (UGT1A1*28) had a common homozygous mutation (HC6/6). The development was favorable after cessation of irinotecan and the resumption of raltitrexed in monotherapy.

In May 2015, due to metastatic lymph node progression, the patient was treated with regorafenib, initially with two 40-mg tablets (3 out of 4 weeks) followed by 3 tablets. No other treatment was administered. Liver biological examinations were normal at the initiation of this treatment and during follow-up every 15 days. At the theoretical day 1 of the third course, when the regorafenib dosage was increased to 4 tablets per day, the patient was hospitalized for jaundice without fever, right hypochondrial pain, diarrhea, and asthenia. An abdominal scan showed no dilation of the intrahepatic bile ducts. A biochemical 


\section{Case Reports in Oncology}

analysis showed acute hepatitis with hepatocellular insufficiency: ALT $=40.5 \times \mathrm{N}$; aspartate aminotransferase $=53.7 \times \mathrm{N}$; total bilirubin $=332 \mu \mathrm{mol} / \mathrm{L}(\mathrm{N}: 3-18$; conjugated $=165 \mu \mathrm{mol} / \mathrm{L}$ and free $=167 \mu \mathrm{mol} / \mathrm{L}$ ); alkaline phosphatases $=1.5 \times \mathrm{N}$; TP $=29 \%$; and factor $\mathrm{V}=69 \%$. The serology for hepatitis A, B, C, and E and human immunodeficiency virus was negative. The polymerase chain reaction for cytomegalovirus, Epstein-Barr virus, herpes simplex virus, parvo virus B19, and varicella zoster virus was negative. Screening was negative for autoimmune hepatitis (antinuclear antibody, smooth muscle antibody, antineutrophil cytoplasmic antibodies, and liver kidney muscle microsomal antibody). A transjugular liver biopsy was performed at day 2 of the onset of hepatocellular failure and showed severe acute hepatitis in the form of necrotic strands associated with portal necrotic inflammatory activity and lymphocyte infiltration (Fig. 1). The clinical and biological evolution was unfavorable until the eventual death of the patient on day 10 .

\section{Discussion}

Regorafenib metabolism is exclusively hepatic, via two enzymes: cytochrome P3A4 (CYP3A4), an important predictor of drug-induced hepatic disease, and UGT1A9 [11-13]. The hepatic disease is even more marked when other CYP3A4-mediated drugs are administered concomitantly [13]. Although UGT1A9 is involved in regorafenib metabolism, its definitive role remains unclear. The UGT1A family has been extensively studied for the predictable toxicity of irinotecan [14]. The active metabolite of irinotecan, SN38, is transformed into an inactive metabolite via glucuronidation. Pharmacokinetic interactions are likely, since regorafenib is a potent inhibitor of UGT1A1 $[13,15]$ and could be metabolized by UGT1A9, as is the case with sorafenib $[13,16]$. Observations of acute hepatocellular insufficiency have been reported in 2 patients who previously had toxicity to irinotecan [12]. Nevertheless, according to our observation, the existence of a 6/6 homozygous common mutation of UGT1A1 does not argue in favor of a likely cross-toxicity with irinotecan. The definitive role of the UGT1A polymorphisms remains to be clarified. The therapeutic combinations FOLFIRI/regorafenib, currently under evaluation [15], will impose a more extensive determination of the UGT1A profile at the start of treatment.

Other potential mechanisms have also been suggested, such as the impact of regorafenib on altered hepatic blood flow [11], probably via sinusoidal obstruction syndrome [17]. An increase in serum lactate dehydrogenase levels indicates hepatic hypoxia and could constitute a biological monitoring parameter during treatment with regorafenib [17]. An autoimmune mechanism was also suggested, highlighting the value of immunosuppressive therapy in addition to discontinuation of regorafenib [18].

Regorafenib is often prescribed to patients already heavily pretreated for metastatic colorectal cancer. The early hepatic toxicity profiles noted in a phase I trial [3] and during subsequent trials $[1,2,9,10]$ led to recommendations for monitoring liver biology every 15 days during the first 2 months of treatment [19]. Hepatic damage is characterized by cytolysis syndrome followed by a secondary increase in bilirubin. In our case, hepatocellular insufficiency also occurred at a subtherapeutic dosage and without any liver metastasis, justifying a very rigorous monitoring of liver parameters in all cases. 


\section{Statement of Ethics}

The authors have no ethical conflicts to disclose.

\section{Disclosure Statement}

The authors have no conflicts of interest to declare.

\section{References}

1 Grothey A, Van Cutsem E, Sobrero A, Siena S, Falcone A, Ychou M, et al: Regorafenib monotherapy for previously treated metastatic colorectal cancer (CORRECT): an international, multicentre, randomised, placebo-controlled, phase 3 trial. Lancet 2013;381:303-312.

-2 Demetri GD, Reichardt P, Kang YK, Blay JY, Rutkowski P, Gelderblom H, et al: Efficacy and safety of regorafenib for advanced gastrointestinal stromal tumours after failure of imatinib and sunitinib (GRID): an international, multicentre, placebo-controlled, phase 3 trial. Lancet 2013;381:295-302. Strumberg D, Scheulen ME, Schultheis B, Richly H, Frost A, Büchert M, et al: Regorafenib (BAY 73-4506) in advanced colorectal cancer: a phase I study. Br J Cancer 2012;106:1722-1727.

4 Teo YL, Ho HK, Chan A: Risk of tyrosine kinase inhibitors-induced hepatotoxicity in cancer patients: a meta-analysis. Cancer Treat Rev 2013;39:199-206.

-5 Iacovelli R, Palazzo A, Procopio G, Santoni M, Trenta P, De Benedetto A, et al: Incidence and relative risk of hepatic toxicity in patients treated with anti-angiogenic tyrosine kinase inhibitors for malignancy. $\mathrm{Br}$ J Clin Pharmacol 2013;77:929-938.

-6 Shah RR, Morganroth J, Shah DR: Hepatotoxicity of tyrosine kinase inhibitors: clinical and regulatory perspectives. Drug Saf 2013;36:491-503.

7 De Wit M, Boers-Doets CB, Saettini A, Vermeersch K, Roncero de Juan C, Ouwerkerk J, et al: Prevention and management of adverse events related to regorafenib. Support Care Cancer 2014;22:837-846.

8 Krishnamoorthy SK, Relias N, Sebastian S, Jayaraman V, Saif MW: Management of regorafenib-related toxicities: a review. Therap Adv Gastroenterol 2015;8:285-297.

-9 Li J, Qin S, Xu R, Yau TC, Ma B, Pan H, et al: Regorafenib plus best supportive care versus placebo plus best supportive care in Asian patients with previously treated metastatic cancer (CONCUR): a randomised, double-blind, placebo-controlled, phase 3 trial. Lancet Oncol 2015;16:619-629.

-10 Yoshino T, Komatsu Y, Yamada Y, Yamazaki K, Tsuji A, Ura T, et al: Randomized phase III trial of regorafenib in metastatic colorectal cancer: analysis of the CORRECT Japanese and non-Japanese subpopulations. Invest New Drugs 2015;33:740-750.

-11 Akamine T, Anda K, Oki E, Saeki H, Nakashima Y, Imamura Y, et al: Acute liver failure due to regorafenib may be caused by impaired liver blood flow: a case report. Anticancer Res 2015;35:4037-4042.

12 Sacré A, Lanthier N, Dano H, Aydin S, Leggenhager D, Weber A, et al: Regorafenib induced severe toxic hepatitis: characterization and discussion. Liver Int 2016;36:1590-1594.

13 Tlemsani C, Huillard O, Arrondeau J, Boudou-Rouquette P, Cessot A, Blanchet B, et al: Effect of glucuronidation on transport and tissue accumulation of tyrosine kinase inhibitors: consequences for the clinical management of sorafenib and regorafenib. Expert Opin Drug Metab Toxicol 2015;11:785794.

14 Cecchin E, Innocenti F, D'Andrea M, Corona G, De Mattia E, Biason P, et al: Predictive role of the UGT1A1, UGT1A7 and UGT1A9 genetic variants and their haplotypes on the outcome of metastatic colorectal cancer patients treated with fluorouracil, leucovorin and irinotecan. J Clin Oncol 2009;27:2457-2465.

15 Schultheis B, Folprecht G, Kuhlmann J, Ehrenberg R, Hacker UT, Köhne CH, et al: Regorafenib in combination with FOLFOX or FOLFIRI as first- or second-line treatment of colorectal cancer: results of a multicenter, phase Ib study. Ann Oncol 2013;24:1560-1567.

16 Peer CJ, Sissung TM, Kim AR, Jain L, Woo S, Gardner ER, et al: Sorafenib is an inhibitor of UGT1A1 but is metabolized by UGT1A9: implications of genetic variants on pharmacokinetics and hyperbilirubinemia. Clin Cancer 2012;18:2099-2107.

17 Takahashi M, Harada S, Suzuki H, Yamashita N, Orita H, Kato M, et al: Regorafenib could cause sinusoidal obstruction syndrome. J Gastrointest Oncol 2016;7:E41-E44.

18 Kuwayama M, Uchino K, Takayoshi K, Komoda M, Kohjima M, Nakamuta M, et al: Immunosuppressant therapy successfully improved regorafenib-induced severe hepatic injury in a patient with metastatic gastrointestinal tumor: a case report. Oncol Lett 2016;11:85-88. 


\section{Case Reports in Oncology}

Béchade et al.: Fatal Acute Liver Failure as a Consequence of Regorafenib Treatment in Metastatic Colon Cancer

19 Sastre J, Argilés G, Benavides M, Feliú J, García-Alfonso P, García-Carbonero R, et al: Clinical management of regorafenib in the treatment of patients with advanced colorectal cancer. Clin Transl Oncol 2014;16:942-953.
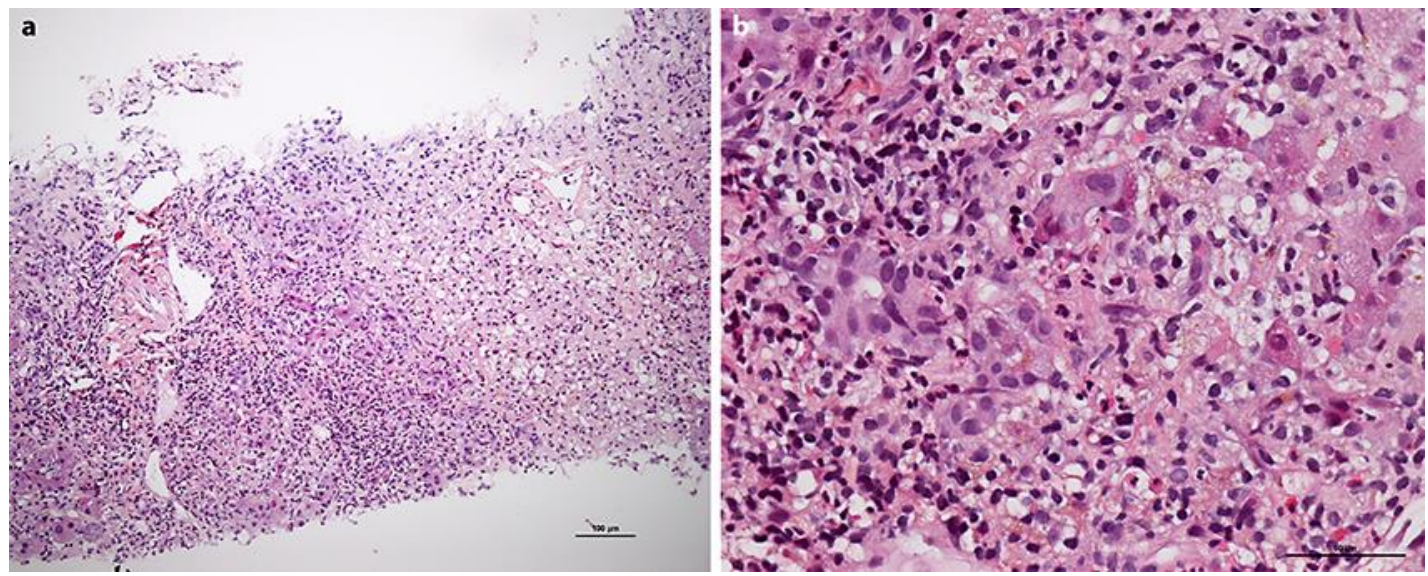

Fig. 1. Severe acute hepatitis with necrotic stands evaluated at $30 \%$ of the parenchyma. HES, $\times 100$ (a) and $\times 400$ (b). 